\title{
Mobile Interfaces for Better Living: Supporting Awareness in a Smart Home Environment
}

\author{
Denis Gračanin ${ }^{1}$, D. Scott McCrickard ${ }^{1}$, Arthur Billingsley ${ }^{2}$, Roosevelt Cooper ${ }^{2}$, \\ Tavon Gatling ${ }^{2}$, Erik J. Irvin-Williams ${ }^{2}$, Felicia Osborne ${ }^{2}$ and Felicia Doswell ${ }^{2}$, \\ ${ }^{1}$ Virginia Tech, Department of Computer Science, 2202 Kraft Drive, \\ Blacksburg, VA 24060, United States \\ ${ }^{2}$ Norfolk State University, Department of Computer Science, 700 Park Avenue, \\ Norfolk, VA 23504, United States \\ \{gracanin, mccricks\}@cs.vt.edu, \{a.b.billingsley,r.d.cooper,t.d.gatling\}@spartans.nsu.edu, \\ erik.taichou@gmail.com,f.d.osborne@spartans.nsu.edu,fdoswell@nsu.edu
}

\begin{abstract}
This paper describes efforts toward creating an integrated living space to support heightened awareness of a user's environment. The work seeks to balance the needs and desires of an individual with those of other people within the locality, community, and world, to include basic comforts like temperature and humidity of a living environment as well as use of resources like power and water. The use of mobile technologies-already prominent among many populations - can be used to raise awareness of the needs and responsibilities of the individual and can highlight opportunities to live in ways that are friendlier to others. Mobile technologies have great promise in connecting users to their environment, and a smart environment enhanced with technology that supports better living can improve the lives of individuals, groups, and the broader community. The goals of this work are toward encouraging: 1) increased awareness of information in the user's surroundings; 2 ) integrated control over factors in one's surrounding and home environments; and 3) increased ability to support sustainable living for both individuals and groups. This work builds on the many smart, green, and sustainable living environment initiatives that have emerged in recent years.
\end{abstract}

Keywords: smart house, awareness, mobile computing.

\section{Introduction}

The notion of a smart home, aware home, or green home has been explored toward incorporating environmentally friendly initiatives and techniques into the built environment. Often computing technology plays a key role in monitoring the information generated by these homes and in controlling certain aspects of the home. Awareness can be raised through the use of mobile notification systems, which attempt to deliver current, important information to the user in an efficient and effective manner. This work first seeks to present key HCI-related questions regarding the presentation of information via mobile device-enabling individuals to 
understand how their decisions may impact the environment, and enabling groups to reach consensus on decisions.

To demonstrate this type and access to information, this work extends our prior experiences with LumenHaus [1], Virginia Tech's award winning solar house at the 2010 European Solar Decathlon competition. This paper outlines an interface construction effort that aggregates weather data. The interface seeks to provide the information such that users can maintain constant knowledge of changing weather patterns, toward making informed decisions about the state of LumenHaus; e.g., whether to vent with outside air, how to position the solar panels, and how to maximize comfort for a diverse group of people in LumenHaus.

The interface is used to investigate emerging interface construction directions through a stakeholder investigation study, conducted with individuals or small groups of architects, technologists, and target users. The study took place via in-house presentations or post-demo symposium discussions, when the participants could understand both the interface and the environment. This study highlighted the needs of the stakeholders, and the results from the study influenced not only the construction of the prototype, but, more importantly, the brainstorming of future directions for mobile device development presented in the next section.

\section{Related Work}

The notion of a smart home, aware home, or green home has been explored at places like Virginia Tech, Georgia Tech [2], Stanford, Florida, and Duke, toward incorporating environmentally friendly initiatives and techniques into the built environment. Often computing technology plays a key role in monitoring the information generated by these homes and in controlling certain aspects of the home. For example, the Georgia Tech Aware Home includes motion sensors that monitor the activities of elderly home dwellers and conveys an overview of the activity level to a remote caretaker [3], and under-development interfaces envision in-home sensors like smart scales and glucometers sharing health related data [4].

Awareness is raised through the use of mobile notification systems. Notification systems attempt to deliver current, important information to the user in an efficient and effective manner. Examples of notification systems include messaging systems, email alerts, and news and stock tickers. Significant development work has taken place in the construction of location-aware notification systems; e.g. [5], [6], [7]. An example of notification interfaces being leveraged in an integrated living space is Intel Research Seattle's UbiFit Garden system, with the central premise to foster more regular physical activity through the use of mobile displays, on-body sensing, and journaling. Results from preliminary field studies have shown UbiFit Garden's effectiveness in helping people maintain a more physically active lifestyle and provide evidence validating the usefulness of interactive technologies such as notification interfaces in supporting health-related behavioral change [8].

Recent technological development has brought us closer towards Weiser's vision of information technology permeating our daily lives [9]. Technology does not only dominate our work lives, but has become an integral part of our domestic lives as 
well. Instead of replacing existing media new technology is adapted into the existing patterns of use [10].

The "smart" house augments the traditional home with a rich computational and communicational infrastructure, such as "smart" devices and sensors that can detect and interact with the inhabitants of the house in novel ways. These augmented devices can improve life in the house, by e.g. providing a richer environment for entertainment and education, as well as simplifying inventory and utility management. For example, the Cyberfridge [11] can detect its contents and allow trusted parties with access to that information from anywhere. Another example is "smart" hot water and heat management based on the preferences of the inhabitants.

There are obvious advantages of the "smart" home outlined above. However, this focus on the domestic also introduces new challenges "...that move design beyond the current focus on information and knowledge work ... and exposes us to the demands of new user groups, including the elderly, the disabled, and the mentally impaired ...." [10].

Furthermore, the home is a nodal point between the public and private [12]. Introducing information technology into that setting, therefore, increases the importance of addressing the enduring concerns towards its effect on privacy and security [13]. These challenges are further exacerbated as both the physical and social structures within the home are subject to continuous change [14], [15]. Thus, adding information technology into our homes has to be done considering the multitude of activities architectural space support [16].

However, this is not limited just to homes. Workplace, public buildings, shops are all architectural spaces where people can interact and perform various tasks. Information technology within the architectural space has to address the social context and our needs for privacy and security in order to offer an experience that is sustainable. While progress is being made addressing these issues, the use of information technology is still far away from being as "refreshing as taking a walk in the woods" [17].

Arguably, the home (especially smart home) is a primary example of humanarchitecture interaction and primary target of current research. We can leverage those results and generalize them to be applicable to a wide variety of architectural spaces. By learning what makes and architectural space a home we can learn about our interaction with architecture in general.

The home is of enormous social meaning [12], and people are selective on what they allow to be brought into their homes [16]. Therefore, any change made to this environment bears consequences. In order to be able to achieve a symbiosis between information technology and the architectural space as a social space, we have to first understand the social context, before introducing new elements into the space.

However, the social intricacies of 'home' are not well understood [10] since the studies into socio-technical context focus more on the workspace than the home [12]. Unfortunately, strategies from the workplace do not translate well into the home [10]. In addition, the studies on the concept of home are more focused on the exotic (e.g. on the tribal home) rather than the mundane [18].

The first thing that needs to be understood is what determines the use of an architectural space, e.g. what makes a house a home, i.e. what distinguishes the home from other places. While the home is a nodal point between the public and private 
[12], it does not only derive its definition from being the opposite of the public space [19]. This distinction is lacking since there are workspaces that are considered private towards outsiders [10].

Crabtree and Rodden [10] study domestic routines as related to communication and collaboration. They introduce three major concepts: ecological habitats as places where communication media live, activity centers as places where media are produced and consumed, and coordinate displays as places where media are made available to coordinate activities.

Aipperspach et al. [16] discuss the dangers of introducing information technology into the home without considering potential detrimental effects on its inhabitants. They suggest the idea of a semi-smart home, where areas are intentionally kept free of technology, except for so-called tourist devices (devices that may visit certain areas, yet have a specific 'home').

This work first seeks to present key CHI-related questions regarding the presentation of information via mobile device-enabling individuals to understand how their decisions may impact the environment, and enabling groups to reach consensus on decisions. We introduce LumenHaus, a self-sustaining home, and describe an initial interface construction effort used to speculate on emerging interface construction directions.

\section{Approach}

Undertaking this effort led to the following questions:

1. What are appropriate methods for collaborative application and social interaction using mobile devices? The growing use of mobile devices, and increased connections to in-home technologies, provides an important opportunity to raise awareness and support interactions for both individuals and groups.

2. What are the constraints and opportunities for mobile new-generation applications? Emerging technologies like the iOS platform (iPhone, iPad, and iPod touch) provide novel interaction techniques - shaking, tilting, multi-touch, tight information integration - that do not yet have accepted uses in the realms of awareness and interaction with data repositories, a focus of this work.

3. How can co-located groups of people effectively balance their needs-and the impact on the environment - toward raising awareness and identifying mutually acceptable decisions? Many decisions regarding an environment are made with immediate impact on a small group of people but environmental impacts on larger groups, as explored in this work.

These questions were explored within the context of a technology-enhanced home that generates data accessible to the iOS platform. Student designers assembled an initial prototype used in participatory design sessions to brainstorm application possibilities. 


\subsection{Designing for LumenHaus}

To explore our questions, we constructed and presented an iPhone interface designed by a group of undergraduate students for LumenHaus (Figure 1). Students visited and worked during the construction of LumenHaus, meeting on multiple occasions with the stakeholder groups, including 1) potential users of the completed structure; 2) architects with experience in constructing LumenHaus and similar facilities; and 3) computer scientists with a good understanding of the feasibility of current and future mobile integrated location-aware devices. These meetings enlightened the students as to the needs of the stakeholders, and the results from the meetings influenced not only the construction of the prototype presented in this section but, more importantly, the brainstorming of future directions for mobile device development.

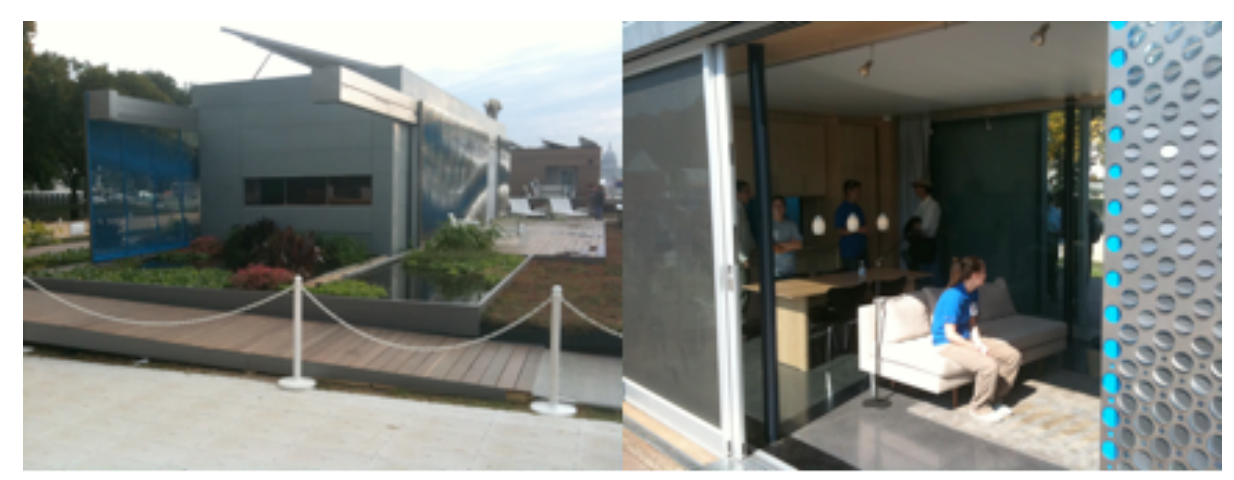

Fig. 1. The LumenHaus [1] energy-efficient living environment. The external view at left shows the movable solar panels and venting system, which can be used to leverage external light and air in a manner optimal for energy usage and human comfort. Similarly, users can view and control water usage, heating, and other in-home aspects. The internal view at right highlights the living environment, which encourages social interaction and benefits from tools for heightened awareness.
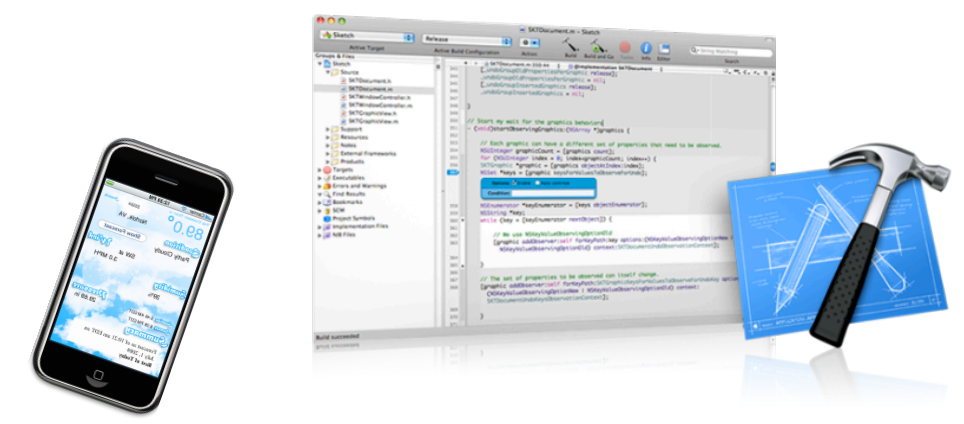

Fig. 2. The development environment.

Students made use of the iOS platform for the development of interfaces. Initial weeks of the project were dedicated to student training session, in which they were 
made familiar with Objective $\mathrm{C}$, the Xcode environment, socket buffering, and the general iPhone programming method (Figure 2). Initial assignments to program image, text, slider, auto-resize on rotation, connection, notification methods, and accelerometer-based interaction provided the programmers with awareness of the display and iteration methods - many of which are unique to mobile platforms like the iPhone. Then, the students built a core application to monitor weather information, intended to connect with LumenHaus.

The weather information display interfaces (Figure 3) provided an aestheticallypleasing, iPhone specific presentation of the local weather at the location of LumenHaus. When the user starts the application, it aggregates web-based weather data, as well as a brief summary for the next few hours, and an extended forecast over several days. The background setting can reflect the external conditions graphicallysunny, cloudy, raining, snowing - so that users can assimilate this more quickly. Simple controls allow users to access and close the application on the move or in group settings with minimal interruption.
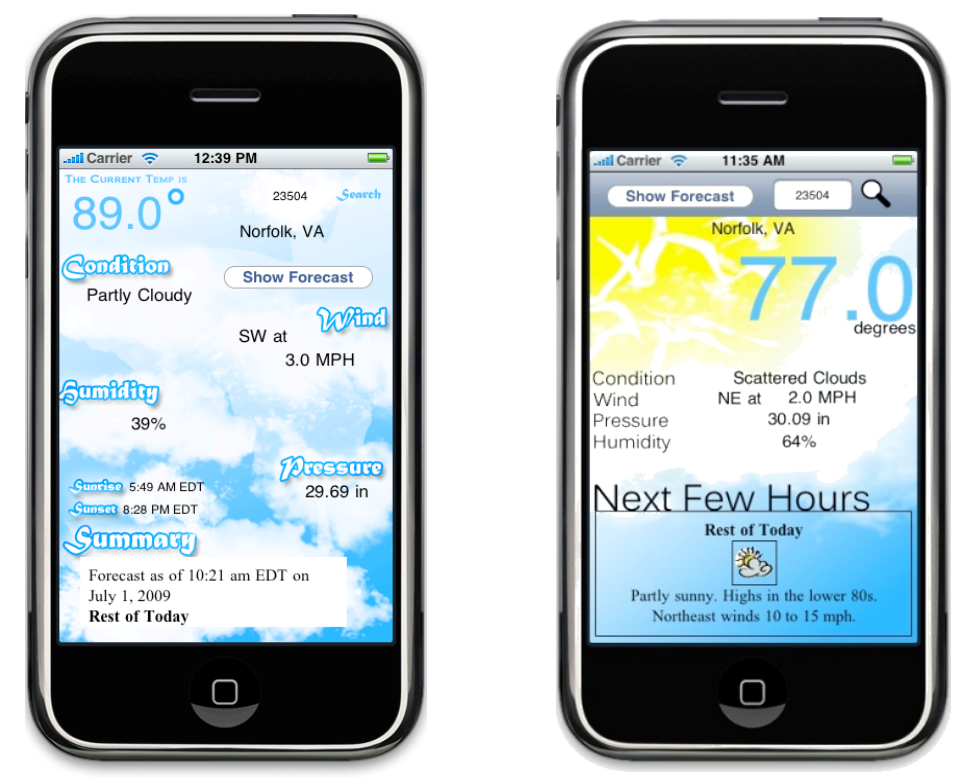

Fig. 3. The LumenHaus weather information displays, highlighting current and forecast local conditions. The visually appealing display shows information of general interest, but also provides opportunities to connect to in-home systems. The left display shows the initial design of the user interface. The right display shows the redesigned user interface based on the feedback and comments provided by the users in the pilot study.

The students then demoed the application to stakeholders in multiple situations: through one-on-one or small group interactions, via in-house presentations, and in an undergraduate research symposium. By making current and projected weather conditions readily available, the students and the stakeholders then brainstormed about potential uses for the information that could be integrated into the display and 
supported via iPhone interaction methods. More broadly, the application provided the opportunity to brainstorm about the other data that is available about the environment-both inside and external to the home-such as lighting conditions, water usage, and internal temperature. Four key classes of interaction methods that emerged from these brainstorming sessions are aggregated and presented Section 4.

\subsection{Evaluation}

The contacted study complements our research on interaction interoperability framework [20], [21] which provides an evaluation testbed for this type of studies.

Initially the application simply connected to wunderground.com and tailored the personalized forecast for iPhone presentation. The revised application code uses peerto-peer based approach to create a Bluetooth data bridge between the host iPhone and a Bluetooth enabled device (i.e. another iPhone or Bluetooth capable device). This is used to transmit the weather data from wunderground.com to the host phone without that device being physically connected to Internet. The result is our LumenWaether application, which provides a web based weather service to the LumenHaus users. Users comments were crucial to this project in evaluating the initial interface (Figure 3 left) and improving it (Figure 3 right).

The pilot study included six users that were chosen were members of the Research Experience for Undergraduates in HCI at Virginia Tech [22]. They were college students who were familiar with using iPhone and similar mobile technology. However, they had no experience in mobile applications design and development.

The users were explained the purpose and features of the application but not how to use it. That was up to the users to discover. The users were then asked to pick the device (iPhone), start the application and explore its features.

They commented that the purpose of the application was not immediately clear. It became apparent that the user interface (Figure 3 left) has to be re-designed to improve clarity of the presented informational. Their comments can be summarized as follows:

- The interface was not sufficiently intuitive. The users were sometimes confused about what to do and how to activate features.

- Data layout and display were not sufficiently well organized. In other words, the information was scattered on the screen and the users were not sure what data was being shown to them.

- The cloud design/background (Figure 3 left) was confusing. The users asked questions like "why are there nothing but clouds?," "Am I supposed to feel like I'm in the sky?".

- Five out of six users did not access all the functionality. They did not realize on their own that they could view an extended daily forecast and seven-day forecast. The one user who did realize and used these features took almost five minutes to do so. 
The received feedback was used to redesign and "cleanup" the user interface (Figure 3 right). The ZIP code selection for the weather information is now positioned at the top of the display area. The current temperature is now more emphasized and clearly visible in the upper right corner of the display area. Some weather data (condition, wind, pressure, humidity) were grouped together and displayed in the middle of the display area. The weather forecast is now clearly displayed at the bottom of the display are. The background image (clouds) was replaced with an "adaptive" image that reflects the current weather situation.

The redesigned user interface (Figure 3 right) addressed the issues discovered in the pilot study and was well accepted by the users.

\section{Conclusions and Future Work}

Leveraging the sample interface designed by the students, the following interface directions emerged.

Home-based interactions provide means to control the display of information from the home, as well as to control aspects of the home environment itself. For example, the students developed a method to shake the display to refresh information about the weather; important in tracking a developing storm or in planning errands. The interfaces should share certain application information with surrounding people running the same application, such as the varying desires for different in-home temperatures or lighting levels - which would impact when and for how long windows would be opened, closed, or shaded. These interaction methods are similar to the ones highlighted earlier for existing smart, green homes (e.g., [3], [4]).

A late joiner display would assist a latecomer to a meeting (either formal or informal) in understanding the major decisions that have been made, toward getting in a position where the latecomer can make meaningful contributions to the group without hindering ongoing group progress. Interface techniques for addressing late joiners have appeared previously (e.g., [23]), with solutions focusing on the aggregation of information and decisions into an easily digestible form and demonstration by the late joiners of an adequate level of understanding. Locationaware technology seems well-suited to advance interface techniques for late joiners, by tracking precisely when a person arrives and leaves (e.g., realizing when a person steps away to the restroom for five minutes.

An annotation tool would support group interaction centered around a shared visual artifact, such as a developing weather report, a home improvement plan, or a shopping list. The tool must support multimedia input (to include typed text, scribbled notes, or brief voice comments) either connected to an artifact or in a standalone form. The explosion of collaborative off-the-desktop interfaces suggests a need for techniques for annotating and, perhaps more importantly, connecting and maintaining the annotations throughout the duration of the course of the interaction time period. The knowledge map that emerges can be viewed either temporally or topically, depending on the preference of the user, and it will support user tagging to facilitate text searching. We seek to make obvious the ability to annotate at any time, through whatever media type and means is most suitable, We anticipate that these interfaces 
(and others like them) will be lightweight bridges to more complete notes, writeups, and reports that are generated, resulting in the added value of an overlaying knowledge annotation map of comments on a collection of more heavyweight documents.

Sustainable consciousness displays would be used to encourage an appropriate balance between individual comfort, group needs, and environmental impact. We envision suggestions regarding energy consumption, lighting methods, and heating/cooling that may change the general lifestyle decisions that users make. For example, instead of turning on a lamp, a person could remove shades and use external light, and instead of air conditioning or heating, a person could make use of differences in internal and external temperatures by venting with outside air. Appropriate presentation of this type of information-with buy-in from all local stakeholders - can lead to sustainable living.

Each of these application areas helps connect individuals and groups to the emerging environments represented by LumenHaus-architectural spaces that provide opportunities to live in a manner better for the individual and the environment. The envisioned tools and applications have promise to connect people to each other, and to raise awareness of the impacts and opportunities of lifestyle choices on the environment.

\section{References}

1. Virginia Tech Lumenhaus, http://www.lumenhaus.com

2. Kidd, C.D., et al.: The Aware Home: A Living Laboratory for Ubiquitous Computing Research. In: 2nd International Workshop on Cooperative Buildings, Integrating Information, Organization, and Architecture, pp. 191--198. Springer-Verlag, London (1999)

3. Rowan, T., Mynatt, E. D.: Digital family portrait field trial: Support for aging in place. In: 23rd SIGCHI Conference on Human Factors in Computing Systems, pp. 521--530 (2005)

4. Medynskiy, Y., Miller, A., Yoo, J.W., Mynatt, E.: Temporal data in a health self management application. In: Interacting with Temporal Data Workshop, 27th International Conference on Human Factors in Computing Systems (2009)

5. LaMarca, A., de Lara, E.: Location Systems: An Introduction to the Technology Behind Location Awareness. Synthesis Lectures on Mobile and Pervasive Computing 3, 1--122 (2008)

6. Sohn, T., et al.: Experiences with place lab: An open source toolkit for location-aware computing. In: 28th international Conference on Software Engineering, pp. 462--471 (2006)

7. Uluca, D., Streefkerk, J.W., Sciacchitano, B., McCrickard, D.S.: Designing automated handheld navigation support. In: 10th International Conference on Human Computer Interaction with Mobile Devices and Services, pp. 481--482 (2008)

8. Consolvo, S., McDonald, D.W., Landay, J.A.: Theory-driven design strategies for technologies that support behavior change in everyday life. In 27th International Conference on Human Factors in Computing Systems, pp. 405--414 (2009)

9. Weiser, M.: The Computer for the 21st Century. Scientific American 265, 94--104 (1991)

10. Crabtree, A., Rodden, T.: Domestic Routines and Design for the Home. Computer Supported Cooperative Work 13, 191--220 (2004) 
11. Mankoff, J., Abowd, G.: Domisilica: Providing Ubiquitous Access to the Home. Technical report, Georgia Institute of Technology (1997)

12. Short, J.R.: Foreword. In: Cieraad, I. (ed.) At Home: An Anthropology of Domestic Space. pp. ix--x. Syracuse University Press, Syracuse, NY (1999)

13. Nissenbaum, H.: Privacy as Contextual Integrity. Washington Law Review 79, 119--158 (2004)

14. Harrison, S., Dourish, P.: Re-place-ing space: the roles of place and space in collaborative systems. In: 1996 ACM conference on Computer Supported Cooperative Work, pp. 67-76. ACM, New York (1996)

15. Rodden, T., Benford, S.: The evolution of buildings and implications for the design of ubiquitous domestic environments. In: 21st SIGCHI conference on Human factors in computing systems, pp 9--16. ACM, New York (2003)

16. Aipperspach, R., Hooker, B., Woodruff, A. The heterogeneous home. Interactions 16, 35-38 (2009)

17. Weiser, M.: The computer for the 21st century. SIGMOBILE Mobile Computing Communication Review 3, 3--11 (1999)

18. Cieraad, I.: Dutch Windows: Female Virtue and Female Vice. In: Cieraad, I. (ed.) At Home: An Anthropology of Domestic Space. pp. 31--51. Syracuse University Press, Syracuse, NY (1999)

19. Cieraad, I.: Introduction. In: Cieraad, I. (ed.) At Home: An Anthropology of Domestic Space. pp. 1--12. Syracuse University Press, Syracuse, NY (1999)

20. Ahmed, H., Gračanin, D.: Context Sensitive Interaction Interoperability for Serious Virtual Worlds. In: Debattista, K., Dickey, M,. Proenca, A., Santos, L. P. (eds.) 2nd International Conference on Games and Virtual Worlds for Serious Applications. pp. 159-$166(2010)$

21. Mulligan, G., Gračanin, D.: A Comparison of SOAP and REST Implementations of a Service Based Interaction Independence Middleware Framework. In: Rossettii, M., Hill, R., Johansson, B. Dunkin, A. (eds.) 2009 Winter Simulation Conference. pp. 1423--1432 (2009)

22. Research Experience for Undergraduates in HCI, Virginia Tech, http://www reu.hci.vt.edu

23. Edwards, W.K., Mynatt, E.D.: Timewarp: Techniques for autonomous collaboration. In: 15th SIGCHI Conference on Human Factors in Computing Systems, pp. 218--225 (1997) 